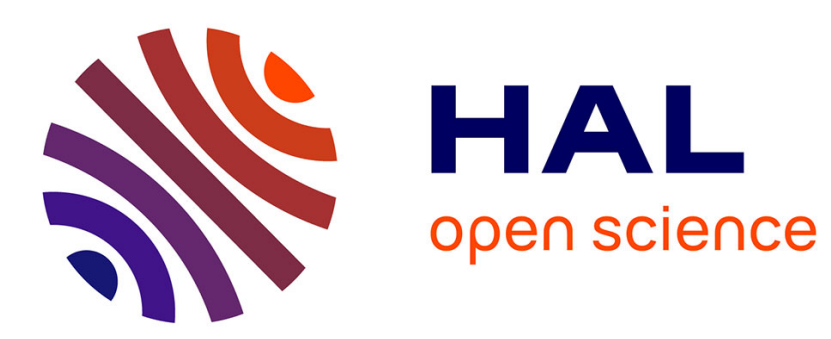

\title{
Exact relation between spatial mean enstrophy and dissipation in confined incompressible flows
}

Florence Raynal

\section{To cite this version:}

Florence Raynal. Exact relation between spatial mean enstrophy and dissipation in confined incompressible flows. Physics of Fluids, 1996, 8 (8), pp.2242-2244. 10.1063/1.868997 . hal-01348399

\author{
HAL Id: hal-01348399 \\ https://hal.science/hal-01348399
}

Submitted on 22 Jul 2016

HAL is a multi-disciplinary open access archive for the deposit and dissemination of scientific research documents, whether they are published or not. The documents may come from teaching and research institutions in France or abroad, or from public or private research centers.
L'archive ouverte pluridisciplinaire HAL, est destinée au dépôt et à la diffusion de documents scientifiques de niveau recherche, publiés ou non, émanant des établissements d'enseignement et de recherche français ou étrangers, des laboratoires publics ou privés. 


\title{
Exact relation between spatial mean enstrophy and dissipation in confined incompressible flows
}

\author{
Florence Raynal \\ Laboratoire de Mécanique des Fluides et d'Acoustique, UMR CNRS 5509, Ecole Centrale de Lyon and \\ Université Lyon I, 36 avenue Guy de Collongue, 69131 Ecully Cédex, France, and \\ Laboratoire de Physique, URA CNRS 1325, ENS Lyon, 46 allée d'Italie, 69364 Lyon Cédex 07, France
}

(Received 23 January 1996; accepted 15 April 1996)

\begin{abstract}
An exact non-trivial relation between spatial mean enstrophy and dissipation in closed incompressible flows is derived analytically, which also applies to unbounded fluid domains when the velocity decays to zero sufficiently rapidly at infinity. The quantity $\left\langle\omega^{2}-\sigma^{2}\right\rangle$ is shown to depend only on the boundary conditions, and it is pointed out that in many confined flows, the equality $\left\langle\omega^{2}\right\rangle=\left\langle\sigma^{2}\right\rangle$ is not satisfied. (C) 1996 American Institute of Physics.
\end{abstract}

[S1070-6631(96)01308-6]

It is now well known ${ }^{1}$ that in a constant density, constant viscosity fluid, by taking the divergence of the Navier-Stokes equations, one finds

$$
\nabla^{2} p=(\rho / 2)\left(\omega^{2}-\sigma^{2}\right),
$$

where $\omega^{2}=(\nabla \wedge \vec{v})^{2}=2 \omega_{i j}^{2}$ is the square of the vorticity modulus, also called enstrophy, and $\sigma^{2}=2 \sigma_{i j}^{2}$ is the square of the rate of strain tensor, with $\omega_{i j}$ and $\sigma_{i j}$ defined as

$$
\begin{gathered}
\omega_{i j}=\frac{1}{2}\left(\partial_{i} v_{j}-\partial_{j} v_{i}\right), \\
\sigma_{i j}=\frac{1}{2}\left(\partial_{i} v_{j}+\partial_{j} v_{i}\right) .
\end{gathered}
$$

Equation (1) is analogous to Poisson's law in electrostatics on the electrical potential, so that $\omega^{2}$ and $\sigma^{2}$ behave, respectively, like negative and positive charges. This simple remark has had a renewal of interest those last years in the field of turbulence, with the direct and numerical observation of vorticity filaments ${ }^{2-8}$ which create intermittency. These filaments have also been characterized by low-pressure events, both experimentally and numerically. ${ }^{9-11}$ It has been emphasized that the fluid is a "neutral" medium, i.e. $\left\langle\omega^{2}\right\rangle$ $=\left\langle\sigma^{2}\right\rangle$, where $\langle X\rangle$ denotes the volume integral $\int_{\mathscr{Q}} X d \tau$. We prove in this paper that the hypothesis of neutrality is not verified for many boundary conditions.

We suppose that the fluid domain $\mathscr{D}$ is either infinite, with a velocity field decaying to zero sufficiently rapidly at infinity (see later), or finite with solid boundaries, in such a case we denote by $V_{f}$ the volume of fluid. The flow might be produced by a solid body $\mathscr{D}_{s}$ moving in the fluid, for instance a body in translation, or a rotating disk. We denote by $V_{s}$ the solid volume. If the domain is finite, the total volume inside the container is $V_{c}=V_{s}+V_{f}$.

Since the velocity field is generally not known exactly (especially in turbulent flows), we have to calculate the quantity $\left\langle\omega^{2}-\sigma^{2}\right\rangle$ indirectly. First of all, we turn the volume integral into a surface integral, keeping in mind that in an incompressible flow, the velocity field satisfies the continuity equation $\partial_{i} v_{i}=0$. We get

$$
\left\langle\omega^{2}-\sigma^{2}\right\rangle=-2 \int_{\mathscr{D}} \partial_{i}\left(v_{j} \partial_{j} v_{i}\right) d \tau=-2 \int_{\Sigma}\left(v_{j} \partial_{j} v_{i}\right) n_{i} d \Sigma,
$$

where $\vec{n}$ is the unit normal to the element of surface, and oriented outward the fluid, as shown in Fig. 1. $\Sigma=\Sigma_{s} \cup \Sigma_{c}$ designates both the surface of the solid, $\Sigma_{s}$, and that of the container, $\Sigma_{c}$, which is eventually at infinity. In this latter case, we suppose that $\vec{v}$ decays to zero like $R^{-\alpha}$ when $R \rightarrow \infty$. A straightforward calculation shows that the surface integral tends to zero at infinity if $\alpha>1 / 2$, which seems to be generally the case in flows produced by a finite moving body. Note again that this result would not hold in open flows like for example pipe flows, since the stress boundary conditions up- and downstream (corresponding to inlet and exit) are not known a priori.

Due to viscosity, the velocity in the fluid at the boundary is equal to the velocity of the solid. However, a problem arises concerning the continuity of the derivatives of the velocity, which is not satisfied a priori. We can nevertheless prove the continuity of $\left(v_{j} \partial_{j} v_{i}\right) n_{i}$ across the solid boundary $\Sigma$.

First of all, we note that this quantity may be written:

$$
\left(v_{j} \partial_{j} v_{i}\right) n_{i}=v_{j} \partial_{j}\left(v_{i} n_{i}\right)-v_{i} v_{j} \partial_{j} n_{i} .
$$

The first term on the right hand-side of the above equation involves the derivatives of the normal velocity, while the second term is related to the curvature of the boundary. The continuity of the velocity field is written as

$$
\vec{v}_{f}(M, t)=\vec{v}_{b}(M, t),
$$

where $M$ is any point on the solid surface $\Sigma$, and the subscripts $f$ or $b$ are used, respectively, to denote a quantity calculated in the fluid domain or in the boundary. Equation (6) immediately implies that at the boundary

$$
\left(v_{i} v_{j} \partial_{j} n_{i}\right)_{f}=\left(v_{i} v_{j} \partial_{j} n_{i}\right)_{b} .
$$

In order to evaluate the first term in the right hand-side of Equation (5), we consider a point $M$ on the boundary and we denote by $\vec{n} \equiv \vec{e}_{z}$ the unit normal at point $M$. The local elementary surface satisfies an equation $z=\xi(x, y)$, with $\xi(0,0)=0$ at point $M$. Equation (6) locally is written as: 


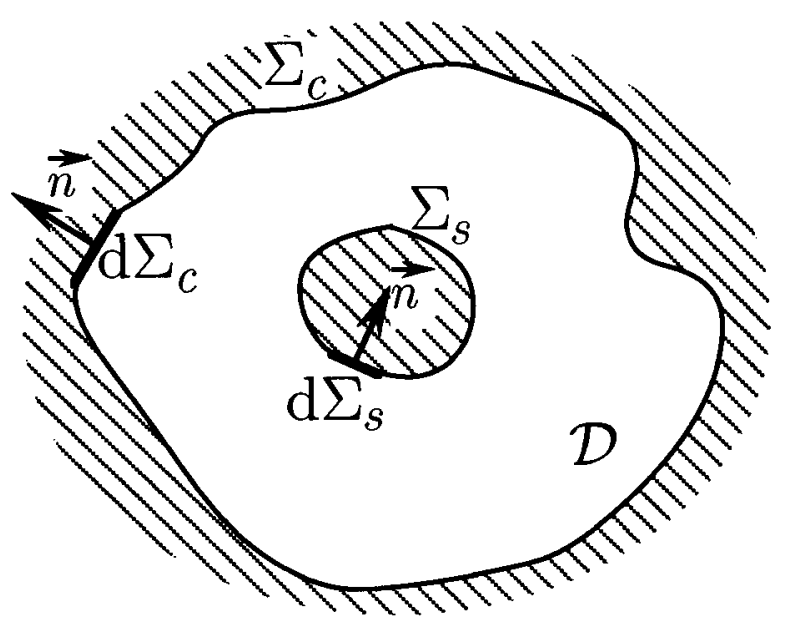

FIG. 1. Orientation of the unit normal $\vec{n}$.

$$
\vec{v}_{f}(x, y, \xi(x, y), t)=\vec{v}_{b}(x, y, \xi(x, y), t) .
$$

However, due to the smoothness of the boundary, the partial derivatives of $\xi$ verify $\left.\partial_{x} \xi\right|_{(0,0)}=\left.\partial_{y} \xi\right|_{(0,0)}=0$ (the surface is locally tangent to a plane), and a Taylor development gives at first order

$$
\begin{aligned}
& \partial_{x} \vec{v}_{f}=\partial_{x} \vec{v}_{b}, \\
& \partial_{y} \vec{v}_{f}=\partial_{y} \vec{v}_{b},
\end{aligned}
$$

at point $M$. Now, because of the continuity equation $\partial_{i} v_{i}=0$, we can write that

$$
\left(v_{j} \partial_{j} v_{z}\right)_{f}=\left(v_{x} \partial_{x} v_{z}+v_{y} \partial_{y} v_{z}-v_{z}\left(\partial_{x} v_{x}+\partial_{y} v_{y}\right)\right)_{f} .
$$

Then, with Equations (8), (9), and (10)

$$
\left(v_{j} \partial_{j} v_{z}\right)_{f}=\left(v_{x} \partial_{x} v_{z}+v_{y} \partial_{y} v_{z}-v_{z}\left(\partial_{x} v_{x}+\partial_{y} v_{y}\right)\right)_{b} .
$$

Note that $v_{i} n_{i}=v_{z}$ is not necessarily null in the general point of view chosen. Now, we use the fact that the incompressibility hypothesis $\partial_{i} v_{i}=0$ is also valid in the solid, and

$$
\left(v_{j} \partial_{j} v_{z}\right)_{f}=\left(v_{j} \partial_{j} v_{z}\right)_{b},
$$

which holds for any point $M$ on the boundary. Together with Equations (5) and (7), we obtain

$$
\int_{\Sigma}\left(v_{j} \partial_{j} v_{i}\right)_{f} n_{i} d \Sigma=\int_{\Sigma}\left(v_{j} \partial_{j} v_{i}\right)_{b} n_{i} d \Sigma .
$$

In the solid boundary, $\sigma_{i j}=0$, and $\nabla \wedge \vec{v}=2 \vec{\Omega}$, where $\vec{\Omega}=\vec{\Omega}(t)$ is the instantaneous rotation rate vector of the solid. We denote $\vec{\Omega}=\vec{\Omega}_{c}$ for the container and $\vec{\Omega}=\vec{\Omega}_{s}$ for the solid body. We drop the subscript $b$ in those calculations. Thus we have

$$
\partial_{j} v_{i}=\omega_{j i}=\frac{1}{2} \varepsilon_{j i k}(\nabla \wedge \vec{v})_{k}=\varepsilon_{j i k} \Omega_{k},
$$

and we finally obtain $v_{j} \partial_{j} v_{i}=(\vec{\Omega} \wedge \vec{v})_{i}$. We can now turn back the surface integral into a volume integral:

$$
\begin{aligned}
& \int_{\Sigma}\left(v_{j} \partial_{j} v_{i}\right)_{b} n_{i} d \Sigma \\
& =\int_{\Sigma_{c}}\left(\vec{\Omega}_{c} \wedge \vec{v}_{c}\right) \cdot \vec{n} d \Sigma+\int_{\Sigma_{s}}\left(\vec{\Omega}_{s} \wedge \vec{v}_{s}\right) \cdot \vec{n} d \Sigma \\
& =\int_{\mathscr{D} \cup \mathscr{D}_{s}} \nabla \cdot\left(\vec{\Omega}_{c} \wedge \vec{v}_{c}\right) d \tau-\int_{\mathscr{D}_{s}} \nabla \cdot\left(\vec{\Omega}_{s} \wedge \vec{v}_{s}\right) d \tau
\end{aligned}
$$

where the sign - is used because $\vec{n}$ is oriented inward the solid on $\Sigma_{s}$ (see Fig. 1). Noting that in a solid

$$
\nabla \cdot(\vec{\Omega} \wedge \vec{v})=\vec{v} \cdot(\nabla \wedge \vec{\Omega})-\vec{\Omega} \cdot(\nabla \wedge \vec{v})=-2 \Omega^{2},
$$

we get

$$
\int_{\Sigma}\left(v_{j} \partial_{j} v_{i}\right)_{b} n_{i} d \Sigma=2\left(V_{s} \Omega_{s}^{2}-V_{c} \Omega_{c}^{2}\right),
$$

and finally, from Equations (4), (14), and (19):

$$
\left\langle\omega^{2}-\sigma^{2}\right\rangle=4\left(V_{c} \Omega_{c}^{2}-V_{s} \Omega_{s}^{2}\right) .
$$

The case of an unbounded domain with velocity decaying to zero more rapidly than $R^{-1 / 2}$ at infinity is recovered by letting $\vec{\Omega}_{c}=\overrightarrow{0}$. Equality (20) proves that $\left\langle\omega^{2}\right\rangle \neq\left\langle\sigma^{2}\right\rangle$ in confined flows produced by rotating solids. Moreover, the quantity $\left\langle\omega^{2}-\sigma^{2}\right\rangle$ does not depend on the dynamics of the flow (laminar or turbulent), but is fixed by the boundary conditions. If $\vec{\Omega}_{c}=\vec{\Omega}_{c}(t)$ or $\vec{\Omega}_{s}=\vec{\Omega}_{s}(t)$, thus $\left\langle\omega^{2}-\sigma^{2}\right\rangle$ also depends on time. We can introduce the local dissipation rate per mass unit, $\epsilon=\nu \sigma^{2}$, where $\nu$ is the kinematic viscosity of the fluid. Thus equation (20) implies

$$
\left\langle\omega^{2}\right\rangle-\left\langle\frac{\epsilon}{\nu}\right\rangle=4\left(V_{c} \Omega_{c}^{2}-V_{s} \Omega_{s}^{2}\right) .
$$

We can discuss some particular cases

(1) $\vec{\Omega}_{s}=\overrightarrow{0}$ and $\vec{\Omega}_{c}=\overrightarrow{0}$ : When the flow is produced by a body having a movement of translation $\left[\vec{\Omega}_{s}=\overrightarrow{0}\right.$ and $\vec{v}(M, t)=\vec{v}(t) \forall M \in \mathscr{D}_{s}$, thus neutrality is rigorously verified, i.e. $\left\langle\omega^{2}\right\rangle=\left\langle\sigma^{2}\right\rangle$. This is the case for example in the experiment by Villermaux et al., ${ }^{8}$ where the flow is produced by an oscillating grid.

(2) $\vec{\Omega}_{s} \neq \overrightarrow{0}$ and $\vec{\Omega}_{c}=\overrightarrow{0}$ : when a solid is rotated in the fluid, then $\left.\left\langle\sigma^{2}\right\rangle\right\rangle\left\langle\omega^{2}\right\rangle$, which corresponds in the analogy to a "positive total charge." This is the case for the example for the flow driven by two counter-rotating cylinders., 2,11 Note however that for a given $\langle\varepsilon\rangle$, although $\langle\vec{\omega}\rangle=\overrightarrow{0}$ [see Equation (28) below], this flow produces less $\left\langle\omega^{2}\right\rangle$ than that studied by Villermaux et al. $^{8}$ When the rotation rate of the disks is constant, Equation (21) may be rewritten:

$$
\left\langle\omega^{2}\right\rangle=\left\langle\frac{\epsilon}{\nu}\right\rangle+C^{s t} .
$$

However, it was shown experimentally for this very flow in air that $\langle\epsilon\rangle$ is a highly time-fluctuating quantity ${ }^{12}$; Hence, because of Equation (22), $\left\langle\omega^{2}\right\rangle$ fluctuates correspondingly. These fluctuations of the dissipation could thus be related to the appearance of the vorticity filaments. 
(3) $\vec{\Omega}_{s}=\overrightarrow{0}$ and $\vec{\Omega}_{c} \neq \overrightarrow{0}$ : in that case, the flow is submitted to a solid body rotation, which creates an additional $\left\langle\omega^{2}\right\rangle$ compared to $\left\langle\sigma^{2}\right\rangle$; in terms of the electrostatic analogy, the domain is "charged negatively."

We are now going to prove in the general case $\vec{\Omega}_{s} \neq 0$ and $\vec{\Omega}_{c} \neq \overrightarrow{0}$ that, in the frame $\mathscr{R}^{\prime}$ of the container, rotating with angular velocity $\vec{\Omega}_{c}, \int_{\mathscr{D}_{c}}\left(\omega^{\prime 2}-2{\sigma^{\prime}}^{2}\right) d \tau=0$. In this frame, $\sigma^{2}=\sigma^{\prime 2}$ is unchanged, and $\vec{\omega}=\vec{\omega}^{\prime}+2 \vec{\Omega}_{c}$. Thus

$$
\int_{\mathscr{D}_{c}} \omega^{2} d \tau=\int_{\mathscr{Q}_{c}} \omega^{\prime 2} d \tau+4 \vec{\Omega}_{c} \cdot \int_{\mathscr{Q}_{c}} \vec{\omega}^{\prime} d \tau+4 V_{c} \Omega_{c}^{2} .
$$

First of all, we calculate $\int_{\mathscr{D}_{c}} \vec{\omega}^{\prime} d \tau$. Noting that, for any fixed vector $\vec{e}$ :

$$
\begin{aligned}
\vec{e} \cdot \int_{\mathscr{D}} \vec{\omega} d \tau & =\int_{\mathscr{D}} \nabla \cdot(\vec{v} \wedge \vec{e}) d \tau \\
& =\int_{\Sigma}(\vec{v} \wedge \vec{e})_{f} \vec{n} d \Sigma=\int_{\Sigma}(\vec{v} \wedge \vec{e})_{b} \vec{n} d \Sigma \\
& =\vec{e} \cdot \int_{\mathscr{D}_{c}} \vec{\omega}_{c} d \tau-\vec{e} \cdot \int_{\mathscr{D}_{s}} \vec{\omega}_{s} d \tau \\
& =\vec{e} \cdot\left(2 V_{c} \vec{\Omega}_{c}-2 V_{s} \vec{\Omega}_{s}\right)
\end{aligned}
$$

we get

$$
\langle\vec{\omega}\rangle=2 V_{c} \vec{\Omega}_{c}-2 V_{s} \vec{\Omega}_{s} .
$$

In the solid body, we have $\sigma_{i j}=0$ and $\vec{\omega}=2 \vec{\Omega}_{s}$. Hence,

$$
\begin{aligned}
\int_{\mathscr{D}_{c}} \vec{\omega}^{\prime} d \tau & =\left\langle\vec{\omega}^{\prime}\right\rangle+\int_{\mathscr{D}_{s}} \vec{\omega}^{\prime} d \tau, \\
& =\langle\vec{\omega}\rangle-2 V_{f} \vec{\Omega}_{c}+2 V_{s}\left(\vec{\Omega}_{s}-\vec{\Omega}_{c}\right)=\overrightarrow{0},
\end{aligned}
$$

because of Equation (28). Thus,

$$
\int_{\mathscr{Q}_{c}}\left(\omega^{\prime 2}-2 \sigma^{\prime 2}\right) d \tau=\int_{\mathscr{Q}_{c}}\left(\omega^{2}-\sigma^{2}\right) d \tau-4 V_{c} \Omega_{c}^{2}=0 .
$$

Therefore neutrality is verified in the frame $\mathscr{B}^{\prime}$ for the domain \{solid+fluid\}: this striking result is simply due to the continuity of $\omega^{2}-\sigma^{2}$ and of its derivatives at the boundaries [Equation (13)], so that the Green-Ostrogradski theorem can be applied directly in $\mathscr{D}_{c}$ for this quantity!

In conclusion, in this paper, we have related the mean enstrophy and dissipation by the formula

$$
\left\langle\omega^{2}-\frac{\varepsilon}{\nu}\right\rangle=4\left(V_{c} \Omega_{c}^{2}-V_{s} \Omega_{s}^{2}\right),
$$

where $V_{c}$ and $V_{s}$ are, respectively, the volume of the container and the volume of the solid, and $\vec{\Omega}_{c}$ and $\vec{\Omega}_{s}$ their instantaneous rotation rates; the case of unbounded flows with velocity decaying to zero more rapidly than $R^{-1 / 2}$ is recovered by letting $\vec{\Omega}_{c}=\overrightarrow{0}$. We assume that this exact result will be of interest for further experiments.

\section{ACKNOWLEDGMENTS}

I wish to thank Thierry Dauxois for helpful comments. I especially thank Stéphan Fauve for many interesting discussions.

${ }^{1}$ P. Bradshaw and Y. M. Khoh, “A note on Poisson's equation for pressure in a turbulent flow,' Phys. Fluids 24, 777 (1981), and references therein. ${ }^{2}$ S. Douady, Y. Couder, and M. E. Brachet, "Direct observations of the intermittency of intense vorticity filaments in turbulence,' Phys. Rev. Lett. 67, 983 (1991).

${ }^{3}$ E. D. Siggia, "Numerical study of small scale intermittency in threedimensional turbulence,', J. Fluid Mech. 107, 375 (1981).

${ }^{4}$ M. E. Brachet, "Géométrie des structures à petite échelle dans le vortex de Taylor-Green,', C. R. Acad. Sci. 311(II), 775 (1990).

${ }^{5}$ Z. S. She, E. Jackson, and S. A. Orszag, "'Intermittent vortex structures in homogeneous isotropic turbulence,'” Nature 344, 226 (1990).

${ }^{6}$ A. Vincent and M. Menneguzi, "The spatial structure and statistical properties of homogeneous turbulence,' J. Fluid Mech. 225, 1 (1991).

${ }^{7}$ J. Jimenez, A. A. Wray, P. Saffman, and R. Rogallo, "The structure of intense vorticity in isotropic turbulence,' J. Fluid Mech. 255, 65 (1992).

${ }^{8}$ E. Villermaux, B. Sixou, and Y. Gagne, "Intense vortical structures in grid-generated turbulence,', Phys. Fluids 7, 2008 (1995).

${ }^{9}$ S. Fauve, C. Laroche, and B. Castaing, "Pressure fluctuations in swirling turbulent flows,' J. Phys. II (France) 3, 271 (1993).

${ }^{10}$ A. Pumir, "A numerical study of pressure fluctuations in threedimensional, incompressible, homogeneous, isotropic turbulence,' Phys. Fluids 6, 2071 (1994)

${ }^{11}$ O. Cadot, S. Douady, and Y. Couder, "Characterization of the lowpressure filaments in a three-dimensional turbulent shear flow,' Phys. Fluids 7, 630 (1995).

${ }^{12}$ R. Labbé, J.-F. Pinton, and S. Fauve, "Power fluctuations in turbulent swirling flows,', J. Phys. II (France), 6, 1 (1996). 